\title{
Postthrombectomy Cerebral Hyperdensity: Usefulness as a Predictor of Future Hemorrhage or Infarction
}

\begin{abstract}
Aim: This study aims to examine the association between the presence of postinterventional cerebral hyperdensities (PCHD) and the occurrence of hemorrhagic transformation. Materials and Methods: We retrospectively analyzed the data of 33 patients who underwent successful mechanical thrombectomy for emergent large vessel occlusion of the anterior circulation and received two computed tomography (CT) scans of the brain; the first (CT1) within $4.5 \mathrm{~h}$ from the procedure and the second (CT2) within 4 days from the procedure. In patients who showed PCHD on CT1, CT2 was checked to detect hemorrhage or infarction in the location of the noted PCHD. Statistical analysis was done through the Fisher's exact test to detect the association between PCHD and hemorrhagic transformation. Results: 28 of the 33 patients $(84.8 \%)$ showed PCHD on CT1. 20 of the 28 patients $(71.4 \%)$ showed infarction on CT2, while 8 patients $(28.6 \%)$ showed hemorrhage. The 5 patients that did not show PCHD on CT1 all showed infarction on CT2. Analysis of our results revealed that although cases with hemorrhage had more frequent positive contrast compared to infarction, this difference did not reach a statistically significant level $(P=0.302)$. Conclusion: We concluded that the presence of PCHD may be associated with future risk of hemorrhagic transformation, but this association did not reach statistical significance. Further studies are needed to validate these results, which may affect the use of antiplatelets in the immediate postthrombectomy period in the presence of PCHD.
\end{abstract}

Keywords: Extravasation, hemorrhage, infarction, staining, thrombectomy

\section{Introduction}

Mechanical thrombectomy is considered the standard of care in patients with acute ischemic stroke due to emergent large vessel occlusion (ELVO) ${ }^{[1]}$ Patients who have undergone mechanical thrombectomy show hyperdense lesions on the treated side on noncontrast computed tomography $(\mathrm{CT})$ done after the procedure in up to $60 \%$ of cases..$^{[2]}$ These hyperdense lesions have been termed as hyperdense areas, ${ }^{[3]}$ contrast staining areas, ${ }^{[4]}$ contrast extravasation, ${ }^{[5]}$ and postinterventional cerebral hyperdensities (PCHD); ${ }^{[6]}$ we will refer to them as PCHD. PCHD has been hypothesized to occur due to disruption of the blood-brain barrier (BBB) by ischemia, which leads to extravasation and trapping of contrast in the ischemic brain tissue. ${ }^{[7-9]}$ The significance of PCHD has been controversial, with studies suggesting that their presence indicates an increased risk of hemorrhagic transformation ${ }^{[3,5,10]}$

This is an open access journal, and articles are distributed under the terms of the Creative Commons Attribution-NonCommercial-ShareAlike 4.0 License, which allows others to remix, tweak, and build upon the work non-commercially, as long as appropriate credit is given and the new creations are licensed under the identical terms.

For reprints contact: reprints@medknow.com and other studies suggesting that their presence is associated with final brain infarction. ${ }^{[4,6,7]}$

In this series, we investigate the significance of PCHD in a cohort of patients who underwent successful mechanical thrombectomy for acute ischemic stroke involving the anterior circulation. Our aim is to identify the significance of presence or absence of PCHD on immediate postthrombectomy CT, specifically whether it could be used as a prognostic factor to predict future hemorrhagic transformation.

\section{Materials and Methods}

\section{Patient selection}

We retrospectively analyzed the data of patients who underwent mechanical thrombectomy for ELVO in the hospitals that were covered by our Acute Stroke team between April 2016 and September 2018. We then included only the patients who had ELVO of the anterior circulation, underwent successful

How to cite this article: Moussa A, El Serwi A, Tork MA. Postthrombectomy cerebral hyperdensity: Usefulness as a predictor of future hemorrhage or infarction. Arab J Intervent Radiol 2020;4:11-5.

\section{Amgad Moussa, Ahmed El Serwi ${ }^{1}$, Mohamed Amir Tork $^{2}$}

Departments of Radiology and ${ }^{2}$ Neurology, Faculty of Medicine, Ain Shams University, ${ }^{1}$ Department of Radiology, Ain Shams University Specialized Hospital, Cairo, Egypt

Received: 18-05-2019 Revised: 22-06-2019 Accepted: 14-07-2019 Published Online: 27-08-2019

Address for correspondence: Dr. Amgad Moussa, 303 East $60^{\text {th }}$ Street, Apt. 24F, New York, NY 10022, USA. E-mail: amgadmoussa89@ gmail.com

Access this article online Website: www.arabjir.com DOI: 10.4103/AJIR.AJIR_8_19 Quick Response Code:

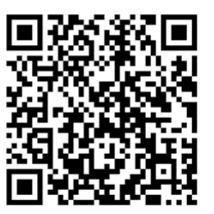


mechanical thrombectomy (TICI 2b/3) in under $6 \mathrm{~h}$ from symptom onset, and received two CT scans of the brain; the first (CT1) within $4.5 \mathrm{~h}$ from the end of the procedure and the second (CT2) within 4 days from the procedure (a minimum of 24-h and a maximum of 4 days' postprocedure). CT1 was checked for the presence or absence of PCHD. In patients who showed PCHD on CT1, CT2 was checked for the presence of hemorrhage or infarction in the location of the noted PCHD. In patients who did not show PCHD on CT1, CT2 was checked for the presence of hemorrhage or infarction on the side of the intervention. The need for informed consent was waived by our institutional review board because we were going through patient data retrospectively.

\section{Mechanical thrombectomy procedure}

All mechanical thrombectomy cases were initiated within $6 \mathrm{~h}$ from symptom onset in patients who had an Alberta Stroke Program Early Computed Tomography Score of $\geq 7$ and done using Solitaire FR Retrievable Stent (Covidien, Ireland). Intravenous tissue plasminogen activator was administered to all eligible patients in accordance with acute ischemic stroke guidelines under the management of the neurology team ( 25 of 33 patients) until time of arterial puncture in the angio suite; however, accurate time and dosage of administered tissue plamsinogen activator (TPA), actilyse (alteplase), boehringer ingelheim, ingelheim, Germany was not recorded. The procedure was done under conscious sedation provided by certified anesthesiologists, and no cases required full anesthesia. Procedure time and amount of contrast used were not recorded for each patient but followed our interventional neuroradiology team guidelines (no upper limit for procedure time or amount of contrast used provided a maximum of 7 stentriever passes was not exceeded until recanalization or aborting the procedure). All other procedure details are beyond the scope of this manuscript. After completion of the procedure, patients were admitted to the intensive care unit for at least $24 \mathrm{~h}$ for close monitoring, which included maintaining blood pressure between $120 / 80 \mathrm{mmHg}$ and $140 / 90 \mathrm{mmHg}$, followed by discharge to a stroke unit.
Imaging analysis

PCHD was defined on CT1 as an area exceeding $0.1 \mathrm{~cm}^{2}$ showing increased density on the side of the intervention with a Hounsfield unit reading at least 10 units higher than the contralateral side, with no surrounding edema and no mass effect. Reading of CT1 was done by two members of the acute stroke team (A.M., A.E.; radiologists with 3 years and 16 years of experience, respectively).

Hemorrhage on CT2 was defined as persistent increased density in the area of previous PCHD, with surrounding edema and with or without mass effect. Infarction on CT2 was defined as decreased density in the area of previous PCHD in comparison to the contralateral side. Reading of CT2 was done by an independent certified radiologist who was blinded to the procedure details and two members of the acute stroke team (A.M., A.E.).

\section{Statistical analysis}

Statistical analysis of our results was done using GraphPad Prism 8 software (California, USA) utilizing the Fisher's exact test for nonparametric comparison of two categorical variables to detect the association between PCHD and hemorrhagic transformation.

\section{Results}

A total of 33 patients fulfilled our inclusion criteria (20 males [60.6\%], 13 females [39.4\%]) with a mean age of 55.2 years (range 21-73 years). Of these patients, $15(45.5 \%)$ had ELVO on the right side and 18 (54.5\%) had ELVO on the left side. 8 patients $(24.2 \%)$ had ELVO of the internal carotid artery and 25 patients $(75.8 \%)$ had ELVO of the middle cerebral artery [Table 1].

Of all the 33 patients who fulfilled our inclusion criteria (irrelevant of the presence or absence of PCHD on CT1), 25 (75.8\%) showed infarction and $8(24.2 \%)$ showed hemorrhage on CT2.

$28(84.8 \%)$ of the 33 patients showed PCHD on CT1 and $5(15.2 \%)$ did not show PCHD on CT1. Of the 28 patients who showed PCHD on CT1, 20 (71.4\%) showed infarction

\begin{tabular}{|c|c|c|c|c|c|c|}
\hline \multirow{3}{*}{$\begin{array}{l}\text { Number (\%) } \\
\text { Gender }\end{array}$} & \multicolumn{2}{|c|}{ Total } & \multirow{2}{*}{\multicolumn{2}{|c|}{$\begin{array}{c}\text { PCHD } \\
28(84.8 \%)\end{array}$}} & \multirow{2}{*}{\multicolumn{2}{|c|}{ No PCHD }} \\
\hline & \multicolumn{2}{|c|}{$33(100 \%)$} & & & & \\
\hline & Males number (\%) & $20(60.6 \%)$ & Males number (\%) & $17(60.7 \%)$ & Males number (\%) & $3(60 \%)$ \\
\hline & Females number (\%) & $13(39.4 \%)$ & Females number (\%) & $11(39.3 \%)$ & Females number $(\%)$ & $2(40 \%)$ \\
\hline \multirow[t]{2}{*}{ Age } & Range years & $21-73$ & Range years & $21-73$ & Range years & $51-64$ \\
\hline & Mean & 55.2 & Mean & 54.6 & Mean & 58.4 \\
\hline \multirow[t]{2}{*}{ ICA } & Right & 2 & Right & 2 & Right & 0 \\
\hline & Left & 6 & Left & 5 & Left & 1 \\
\hline \multirow[t]{2}{*}{ MCA } & Right & 13 & Right & 12 & Right & 1 \\
\hline & Left & 12 & Left & 9 & Left & 3 \\
\hline Infarction & Number (\%) & $25(75.8 \%)$ & Number (\%) & $20(71.4 \%)$ & Number (\%) & $5(100 \%)$ \\
\hline Hemorrhage & Number (\%) & $8(24.2 \%)$ & Number (\%) & $8(28.6 \%)$ & Number (\%) & $0(0 \%)$ \\
\hline
\end{tabular}


and $8(28.6 \%)$ showed hemorrhage on CT2 at the site of PCHD. The 5 patients that did not show PCHD on CT1 all showed infarction on CT2.

Statistical analysis of our results revealed that although cases with hemorrhage had more frequent positive contrast compared to infarction, this difference did not reach a statistically significant level $(P=0.302)$ [Table 2].

\section{Discussion}

Cerebral hyperdensities following intra-arterial therapy for acute ischemic stroke were first reported in 1993 by Komiyama et al. ${ }^{[1]}$ They were initially believed to be a predictor of future hemorrhagic transformation in the era of intra-arterial thrombolytic therapy, ${ }^{[3]}$ but more recent studies support the hypothesis that PCHD is not useful as a predictor for the occurrence of hemorrhagic transformation in the era of mechanical thrombectomy ${ }^{[2,12]}$ [Figure 1].

Parrilla et al. studied 48 patients who underwent mechanical thrombectomy and received two CT scans of the brain; the first within $24 \mathrm{~h}$ and the second between 48 and $72 \mathrm{~h}$ from the intervention. 15 patients showed PCHD on the first CT, 6 of which persisted on the second CT with no reported cases of massive hemorrhagic transformation. The authors concluded that PCHD does not indicate an increased risk of symptomatic hemorrhage. ${ }^{[12]}$

Other authors hypothesized that PCHD was more useful as a predictor of the final infarction size rather than a predictor of hemorrhagic transformation ${ }^{[4,6,7,13]}$ [Figure 2]. Schneider et al. assessed the relationship between preintervention, confirmed infarction seen on diffusion weighted imaging and PCHD in 27 patients who underwent mechanical thrombectomy, and concluded that PCHD represents the minimum final infarct volume at a certain point in time. ${ }^{[13]}$

Some authors argued that PCHD was not one single entity. Yoon et al. studied 62 patients who received intra-arterial

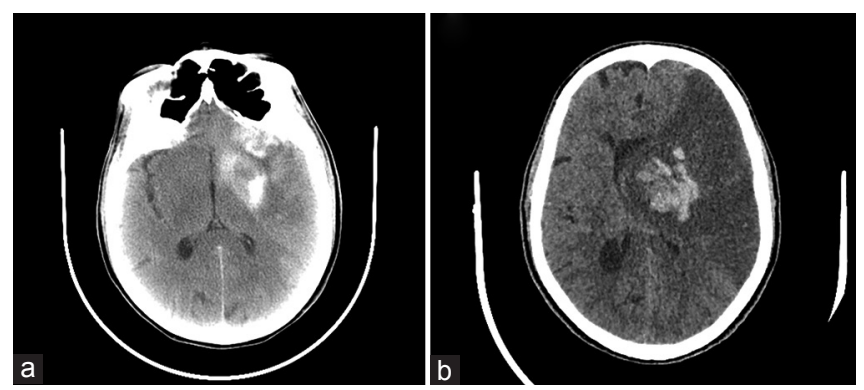

Figure 1: A 63-year-old patient who presented with acute ischemic stroke due to left internal carotid artery occlusion. (a) Nonenhanced computed tomography scan immediately after successful thrombectomy shows hyperdensity in the region of the left lentiform nucleus consistent with postinterventional cerebral hyperdensities. (b) Nonenhanced computed tomography scan two days after thrombectomy (and following deterioration in consciousness level) shows a large area of hemorrhage in the location of the previously noted postinterventional cerebral hyperdensities consistent with hemorrhagic transformation thrombolysis and received two CT scans of the brain; one immediately following the intervention and the other $24 \mathrm{~h}$ after the intervention. They described contrast that appears on the first CT but disappears on the second CT as "contrast enhancement," and contrast that persists on the second CT and shows a Hounsfield unit reading of $>90$ as "contrast extravasation." They noted that patients who showed contrast extravasation had a statistically significant higher incidence of symptomatic hemorrhagic transformation and poor outcome. ${ }^{[5]}$

Contrast extravasation, as defined by Yoon et al., ${ }^{[5]}$ is typically referred to as hemorrhagic transformation by other authors because it goes in concordance with one of the main differentiating points used to differentiate between PCHD and hemorrhage, persistence on follow-up scans. ${ }^{[3]}$ Other factors can be used to differentiate between PCHD and hemorrhagic transformation such as Hounsfield unit reading, ${ }^{[11]}$ morphology of the lesion, ${ }^{[4]}$ dual-energy $\mathrm{CT},{ }^{[14,15]}$ and magnetic resonance imaging ${ }^{[16]}$ but are beyond the scope of this paper. Yoon et al. acknowledge that what they call "contrast extravasation" is a mixture of blood and contrast material, and the increase in the size of the hyperdensity with positive mass effect is what they term symptomatic hemorrhagic transformation. ${ }^{[5]}$ We believe that this is just an increase in the size of a parenchymal hematoma that was present on the first CT.

The pathophysiology of PCHD is closely related to the integrity of the cerebral microvasculature, which is

Table 2: Correlation between postinterventional cerebral hyperdensities and hemorrhage and infarction

\begin{tabular}{|c|c|c|c|c|c|c|}
\hline & \multicolumn{4}{|c|}{ Lesion Type } & \multirow[t]{3}{*}{$P$} & \multirow[t]{3}{*}{ Significance } \\
\hline & \multicolumn{2}{|c|}{ Infarction } & \multicolumn{2}{|c|}{ Hemorrhage } & & \\
\hline & Count & $\begin{array}{c}\text { Column } \\
n \%\end{array}$ & Count & $\begin{array}{c}\text { Column } \\
n \%\end{array}$ & & \\
\hline \multicolumn{7}{|l|}{ Contrast } \\
\hline Positive & 20 & $80 \%$ & 8 & $100 \%$ & 0.302 & Not \\
\hline Negative & 5 & $20 \%$ & 0 & $0 \%$ & & Significant \\
\hline
\end{tabular}
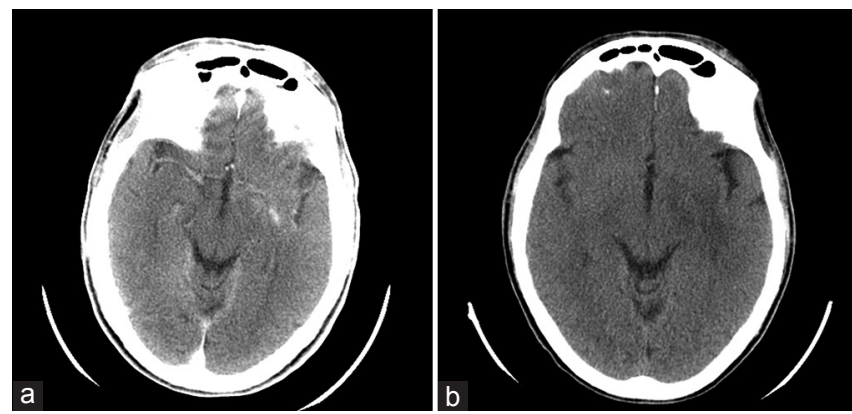

Figure 2: A 61-year-old patient who also presented with acute ischemic stroke due to left internal carotid artery occlusion. (a) Nonenhanced computed tomography scan immediately after successful thrombectomy shows faint hyperdensity in the region of the medial aspect or the left temporal lobe consistent with postinterventional cerebral hyperdensities. (b) Nonenhanced computed tomography scan three days after thrombectomy shows a hypodensity in the location of the previously noted postinterventional cerebral hyperdensities consistent with established ischemic infarction 
maintained by the basal lamina and the BBB, mainly formed by the tight junctions between endothelial cells. ${ }^{[17,18]}$ Ischemia causes gradual breakdown in the basal lamina and is considered the first step in a process that may result in hemorrhagic transformation. ${ }^{[19]}$ Ischemia also leads to increased expression of leukocyte adhesion receptors by endothelial cells, which leads to leukocyte diapedesis and activation, adding to the damage to the BBB and the basal lamina. ${ }^{[20]}$

The damage caused by the ongoing ischemic process is aggravated by the toxic effects of direct intra-arterial injection of contrast media on the cerebral microvasculature, which is believed to occur due to the hyperosmolarity and consequent increased viscosity of contrast media, as well as direct contrast media chemotoxicity. ${ }^{[21-23]}$ Authors have postulated that reperfusion of the cerebral blood vessels following successful recanalization also contributes to damage to the BBB. ${ }^{[24]}$

We hypothesize that all these factors as well as the mechanical trauma caused by the equipment used during mechanical thrombectomy ${ }^{[25]}$ contribute to damage of the BBB and basal lamina, which leads to PCHD, and when combined with other risk factors for hemorrhagic transformation, ${ }^{[26]}$ it may result in hemorrhagic transformation.

Our results add to the studies that concluded that PCHD was not a statistically significant predictor of risk of hemorrhagic transformation. However, our data trends that the absence of PCHD after mechanical thrombectomy for acute ischemic stroke may be a good prognostic factor, though with no statistical evidence likely due to the relatively small number of patients. Of the 5 patients that did not show PCHD, none showed hemorrhagic transformation. Lummel et al. ${ }^{[10]}$ showed that 16 patients $(15.8 \%)$ did not show PCHD of 101 patients who had mechanical recanalization for acute ischemic stroke, which fall in line with our numbers (5 of 33 patients, $15.1 \%$ ), yet differ from other, widely varying, reported numbers $\left(66.1 \%{ }^{[5]}\right.$ and $\left.39.2 \%{ }^{[6]}\right)$. However, Lummel et al. ${ }^{[10]}$ report that 2 of those 16 patients $(12.5 \%)$ developed clinically insignificant hemorrhagic transformation, which differs from our results $(0 \%)$. Other studies also showed varying incidence of hemorrhagic transformation in the absence of PCHD $\left(35 \%,{ }^{[6]} 43.9 \%{ }^{[5]}\right)$.

Limitations to our study include a relatively small sample size, which prevented us from detecting the statistical significance of the absence of PCHD and a possible relationship with consequent absence of hemorrhage.

\section{Conclusion}

Although patients who developed hemorrhagic transformation more often showed PCHD compared to patients who developed ischemic infarctions, this difference did not reach a statistically significant level. Further studies are needed to accurately assess the relation between presence or absence of PCHD and hemorrhagic transformation as well as neurological outcome.

\section{Declaration of patient consent}

The authors certify that they have obtained all appropriate patient consent forms. In the form the patient(s) has/have given his/her/their consent for his/her/their images and other clinical information to be reported in the journal. The patients understand that their names and initials will not be published and due efforts will be made to conceal their identity, but anonymity cannot be guaranteed.

Financial support and sponsorship

Nil.

Conflicts of interest

There are no conflicts of interest.

\section{References}

1. Powers WJ, Rabinstein AA, Ackerson T, Adeoye OM, Bambakidis NC, Becker K, et al. 2018 guidelines for the early management of patients with acute ischemic stroke: A guideline for healthcare professionals from the American Heart Association/ American Stroke Association. Stroke 2018;49:e46-110.

2. Takei J, Irie K, Tanaka T, Okuno K, Hasegawa I, Shimoyama T, et al. Evaluation of the CT high-density area after endovascular treatment for acute ischemic stroke. J Neuroendovasc Ther 2017;11:227-34

3. Nakano S, Iseda T, Kawano H, Yoneyama T, Ikeda T, Wakisaka S, et al. Parenchymal hyperdensity on computed tomography after intra-arterial reperfusion therapy for acute middle cerebral artery occlusion: Incidence and clinical significance. Stroke 2001;32:2042-8.

4. Amans MR, Cooke DL, Vella M, Dowd CF, Halbach VV, Higashida RT, et al. Contrast staining on CT after DSA in ischemic stroke patients progresses to infarction and rarely hemorrhages. Interv Neuroradiol 2014;20:106-15.

5. Yoon W, Seo JJ, Kim JK, Cho KH, Park JG, Kang HK, et al. Contrast enhancement and contrast extravasation on computed tomography after intra-arterial thrombolysis in patients with acute ischemic stroke. Stroke 2004;35:876-81.

6. Nikoubashman O, Reich A, Gindullis M, Frohnhofen K, Pjontek R, Brockmann MA, et al. Clinical significance of post-interventional cerebral hyperdensities after endovascular mechanical thrombectomy in acute ischaemic stroke. Neuroradiology 2014;56:41-50.

7. Cabral FB, Castro-Afonso LH, Nakiri GS, Monsignore LM, Fábio S, Dos Santos AC, et al. Hyper-attenuating brain lesions on CT after ischemic stroke and thrombectomy are associated with final brain infarction. Interv Neuroradiol 2017;23:594-600.

8. Desilles JP, Rouchaud A, Labreuche J, Meseguer E, Laissy JP, Serfaty JM, et al. Blood-brain barrier disruption is associated with increased mortality after endovascular therapy. Neurology 2013;80:844-51.

9. Khatri R, McKinney AM, Swenson B, Janardhan V. Blood-brain barrier, reperfusion injury, and hemorrhagic transformation in acute ischemic stroke. Neurology 2012;79:S52-7.

10. Lummel N, Schulte-Altedorneburg G, Bernau C, Pfefferkorn T, Patzig M, Janssen $\mathrm{H}$, et al. Hyperattenuated intracerebral lesions after mechanical recanalization in acute stroke. AJNR Am J Neuroradiol 2014;35:345-51. 
11. Komiyama M, Nishijima Y, Nishio A, Khosla VK. Extravasation of contrast medium from the lenticulostriate artery following local intracarotid fibrinolysis. Surg Neurol 1993;39:315-9.

12. Parrilla G, García-Villalba B, Espinosa de Rueda M, Zamarro J, Carrión E, Hernández-Fernández $\mathrm{F}$, et al. Hemorrhage/contrast staining areas after mechanical intra-arterial thrombectomy in acute ischemic stroke: Imaging findings and clinical significance. AJNR Am J Neuroradiol 2012;33:1791-6.

13. Schneider T, Mahraun T, Schroeder J, Frölich A, Hoelter P, Wagner $\mathrm{M}$, et al. Intraparenchymal hyperattenuations on flat-panel CT directly after mechanical thrombectomy are restricted to the initial infarct core on diffusion-weighted imaging. Clin Neuroradiol 2018;28:91-7.

14. Tijssen MP, Hofman PA, Stadler AA, van Zwam W, de Graaf R, van Oostenbrugge RJ, et al. The role of dual energy $\mathrm{CT}$ in differentiating between brain haemorrhage and contrast medium after mechanical revascularisation in acute ischaemic stroke. Eur Radiol 2014;24:834-40.

15. Phan CM, Yoo AJ, Hirsch JA, Nogueira RG, Gupta R. Differentiation of hemorrhage from iodinated contrast in different intracranial compartments using dual-energy head CT. AJNR Am J Neuroradiol 2012;33:1088-94.

16. Nikoubashman O, Jablawi F, Dekeyzer S, Oros-Peusquens AM, Abbas Z, Lindemeyer J, et al. MRI appearance of intracerebral iodinated contrast agents: Is it possible to distinguish extravasated contrast agent from hemorrhage? AJNR Am J Neuroradiol 2016;37:1418-21.

17. Hamann GF, Okada Y, del Zoppo GJ. Hemorrhagic transformation and microvascular integrity during focal cerebral ischemia/ reperfusion. J Cereb Blood Flow Metab 1996;16:1373-8.

18. del Zoppo GJ, von Kummer R, Hamann GF. Ischaemic damage of brain microvessels: Inherent risks for thrombolytic treatment in stroke. J Neurol Neurosurg Psychiatry 1998;65:1-9.

19. Hamann GF, Okada Y, Fitridge R, del Zoppo GJ. Microvascular basal lamina antigens disappear during cerebral ischemia and reperfusion. Stroke 1995;26:2120-6.

20. Del Maschio A, Zanetti A, Corada M, Rival Y, Ruco L, Lampugnani MG, et al. Polymorphonuclear leukocyte adhesion triggers the disorganization of endothelial cell-to-cell adherens junctions. J Cell Biol 1996;135:497-510.

21. Wilcox J, Wilson AJ, Evill CA, Sage MR. A comparison of blood-brain barrier disruption by intracarotid iohexol and iodixanol in the rabbit. AJNR Am J Neuroradiol 1987;8:769-72.

22. Kurosawa Y, Lu A, Khatri P, Carrozzella JA, Clark JF, Khoury J, et al. Intra-arterial iodinated radiographic contrast material injection administration in a rat middle cerebral artery occlusion and reperfusion model: Possible effects on intracerebral hemorrhage. Stroke 2010;41:1013-7.

23. Sage MR, Wilcox J, Evill CA, Benness GT. Comparison and evaluation of osmotic blood-brain barrier disruption following intracarotid mannitol and methylglucamine iothalamate. Invest Radiol 1982;17:276-81.

24. Molina CA, Montaner J, Abilleira S, Ibarra B, Romero F, Arenillas JF, et al. Timing of spontaneous recanalization and risk of hemorrhagic transformation in acute cardioembolic stroke. Stroke 2001;32:1079-84.

25. Bourcier R, Saleme S, Labreuche J, Mazighi M, Fahed R, Blanc $\mathrm{R}$, et al. More than three passes of stent retriever is an independent predictor of parenchymal hematoma in acute ischemic stroke. J Neurointerv Surg 2019;11:625-9.

26. Soize S, Barbe C, Kadziolka K, Estrade L, Serre I, Pierot L, et al. Predictive factors of outcome and hemorrhage after acute ischemic stroke treated by mechanical thrombectomy with a stent-retriever. Neuroradiology 2013;55:977-87. 